\title{
Predicting patient nurse-level intensity for a subsequent shift in the intensive care unit: A single-centre prospective observational study
}

KarenDecocka, Michael P.Casaera, FabianGuïzaa, PieterWoutersa, MiekeFlorquina, AlexanderWilmerb, StefanJanssens ${ }^{c}$, SandraVerelst ${ }^{d}$, GreetVan den Berghea, LukBruyneelet

Intensive Care Department, University Hospitals Leuven, Herestraat 49, 3000 Leuven, Belgium

Medical Intensive Care Department, University Hospitals Leuven, Herestraat 49, 3000 Leuven, Belgium

Cardiac Intensive Care, Department of Cardiovascular Diseases, University Hospitals Leuven, Herestraat 49, 3000 Leuven, Belgium

Emergency Department, University Hospitals Leuven, Herestraat 49, 3000 Leuven, Belgium

Leuven Institute for Healthcare Policy, KU Leuven - University of Leuven, Kapucijnenvoer 35, 3000 Leuven

Belgium \& Quality Improvement Department, University Hospitals Leuven, Herestraat 49, 3000 Leuven, Belgium

\section{Abstract}

\section{Background}

A dynamic optimized nurse staffing model for the Intensive Care Unit (ICU), requires a tool for monitoring nurse-level intensity with validated cut-offs to identify patients requiring 1:1, 2:1 or 3:1 patient-to-nurse ratios.

\section{Objectives}

We aimed to determine the Nursing Activities Score (NAS) cut-off values which can best distinguish between high, average and lower nurse-level intensity as unanimously perceived by care providers, and to evaluate whether these NAS cutoffs allow to predict nurse-level intensity in the next shift or the same shift the next day.

\section{Design}


A prospective observational study.

\section{Setting}

9 ICUs in a Belgian tertiary care center.

\section{Participants}

All 3295 patients admitted between March 20, 2013, and September 12, 2013 were included. NAS was quantified at the end of each shift using automatically derived and manually entered care information. Additionally, 412 nurses, 24 nurse managers and 37 physicians rated perceived nurse-level intensity.

\section{Methods}

We first assessed concordance between nurses', nurse managers' and physicians' perceptions of lower (3:1 patient-to-nurse ratio), average (2:1 patient-to-nurse ratio) and high (1:1 patient-to-nurse ratio) nurse-level intensity. Next, receiver operating characteristic (ROC) analysis was applied to determine the NAS cut-offs that best distinguish between different levels of perceived intensity for cases with concordant opinions. Last, logistic regression analysis was applied to estimate the ability of these NAS cut-offs to predict low and high perceived intensity during the next shift and during the same shift the next day.

\section{Results}

Nurses', nurse managers' and physicians' perceptions were concordant in 57.1\% ( $n=4693)$ of cases, mostly concerning lower or average intensity. Optimal NAS cut-offs for lower and high intensity patients equaled $52.7 \%$ and $69.8 \%$, respectively. The lower intensity NAS cut-off showed $74.0 \%$ accuracy to predict lower intensity in the next shift and $75.9 \%$ accuracy to predict lower intensity for the same shift the next day. The high intensity NAS cut-off showed $67.9 \%$ accuracy to predict high intensity in the next shift and $72.0 \%$ accuracy to predict high intensity for the same shift the next day.

\section{Conclusions}


NAS cut-offs could contribute considerably in predicting patient nurse-level intensity, and thus patient-to-nurse staffing ratios, in the next shift or day. Identification or prediction of high intensity, nevertheless, appears most complex and requires further study. Future studies need to account for the many confounding variables which complicate nurse staffing planning.

\section{Keywords}

Intensive care units

Critical care

Nursing activities score

Workload

Statistics \& numerical data

Surveys and questionnaires

Physicians

Nurses

Manpower

What is already known about the topic?

- The Nursing Activities Score allows for the demands of critically ill patients to be assessed on a shift-by-shift basis. The tool has been extensively validated and is used by intensive care unit managers across the globe to make evidence-based staffing decisions.

- The Nursing Activities Score has initially been designed starting from specific nursing activities and summation of time consumed. Less is known about the clinical judgement of patients' nurse-level intensity as a reference to validate the Nursing Activities Score, indicating the patient-to-nurse ratio to provide adequate and safe care.

- Like other nursing workload measurement tools, the Nursing Activities Score relies solely on nurses' reports and perceptions, whereas other health professionals may also contribute to an adequate estimation of the stability of a patient's nurse-level intensity over time.

\section{What this paper adds}

- In this prospective observational study, the subjective appreciation of nurse-level intensity by more than 400 nurses, nurse managers and 
physicians of 8215 patient shifts correlated rather good concerning the large group of normal intensity patient-shifts, but unanimity on high intensity shifts requiring a patient-to-nurse ratio of 1:1 was rare.

- Receiver Operating Characteristics analysis suggested that optimal Nursing Activities Score cut-offs for lower and high intensity patients equaled $52.7 \%$ and $69.8 \%$, respectively.

- These Nursing Activities Score cut-offs contributed considerably in predicting patient intensity both in the immediate following shift as well as in the same shift the next day.

\section{Background}

Providing adequate nurse staffing may be a cost-effective hospital management strategy to improve quality of care and safety. However, cutting expenses at the cost of reduced number of nurses staffed or a downgraded level of skill mix may be detrimental (Rothberg et al., 2005; Needleman et al., 2006; Aiken et al., 2014). This may be particularly true for the Intensive Care Unit (ICU) where increased nurse staffing is associated with reduced mortality, morbidity, and shortened ICUstay in several meta-analyses (Kane et al., 2007; Aragon Penoyer, 2010; Numata et al., 2006; West et al., 2009; McGahan et al., 2012). Previous studies confirmed the aforementioned association particularly in patients with a high risk of death and at a patient-to-nurse ratio exceeding 2.5 patients per nurse. (West et al., 2014; Neuraz et al., 2015). Not only suboptimal nurse staffing, but also increased clinical workload - as reflected by patient turnover and the number of life-sustaining medical procedures - are inversely associated with survival (Neuraz et al., 2015).

Although inherent limitations of these studies, including their retrospective and observational design preclude conclusions about causality, health authorities worldwide did not wait for the ultimate confirmatory evidence to apply or propose mandatory nurse staffing ratios. In the US, several states require by law a maximum of one or two patients per nurse, depending on the type of unit (ICU or Emergency Room (ER)) (Coffman et al., 2002) or on the stability of the patient (American Nurses Association 2015). Similarly, the College of Intensive Care Medicine of Australia and New Zealand defines a minimum standard of 1 nurse per single critically ill patient, and 1 nurse per two lower acuity patients (College of Intensive Care Medicine of Australia and New Zealand 2011). According to Belgian law, two nurses, of which at least one with a special ICU-qualification, 
must be present per six ICU-patients. Furthermore, "the nursing team must adapt to the activities” yet, no further specification is provided (Federal Public Service Health Food Chain Safety and Environment 1998).

As nurse-level intensity varies strongly among ICU patients (Kiekkas et al., 2008), harmonizing available nurse staffing with the actual clinical workload on a day-today basis probably leads to the safest patient care. Therefore, dynamic staff allocation strategies require a valid tool to accurately assess the needs of patient care at any time in the unit. One such tool is the Therapeutic Intervention Scoring System (TISS). This tool originally contained 57 items, but modified versions with 28 items (TISS-28) and 76 items (TISS-76) are used more frequently (Cullen et al., 2020; Keene and Cullen, 1983; Miranda et al., 1996). The latter revealed an inverse association between workload per nurse and risk-adjusted odds of survival (Lee et al., 2017). Although the TISS-28 explains 86\% of the TISS-76 (Miranda et al., 1996), it only accounts for $43 \%$ of nurse workload (Miranda et al., 1996). In contrast, the Nursing Activities Score (NAS) covers 81\% of the actual nurses' activities. This internationally validated tool is based on the TISS-28, enriched with 5 new items and 14 sub-items (Miranda et al., 2003). It is expressed as a percentage between $0 \%$ and $177 \%$, where the volume of nursing care that one nurse can provide during one shift equals 100\% (Miranda et al., 2003). Several studies identified the NAS as the most appropriate tool to evaluate the intensity of care (Padilha et al., 2010; Padilha et al., 2015; Gonçalves et al., 2007; Ferreira et al., 2017; Macedo et al., 2016), and consequently risk of mortality and morbidity (Padilha et al., 2008). A Belgian single-center study revealed that NAS could identify differences in nursing care workload between consecutive shifts, and could therefore be a guide for shift-by-shift staff allocation (Debergh et al., 2012). Consensus-derived NAS cut-off values for differentiating between lower and high nurse-level intensity patients would facilitate the development of a dynamic optimized nurse staffing model. Such cut-offs should go beyond the initial validation based on summation time consumed per nursing act and rely on real-life clinical judgement identifying patients that can be adequately taken care of in the setting of 3:1, 2:1 or even 1:1 patient to nurse ratio. The importance of assessing nurses' work in the ICU through a consensus-driven approach, meaning that both nurses' as well as physicians' views are included, was already recognized in the development of the NAS. Physicians and nurses jointly identified patient- 
condition-related nurse activities that may influence the use of nursing time in the ICU (Miranda et al., 2003).

For the development of such a dynamic staffing model, an adequate estimation of the "stability" of the workload over time in a given patient is crucial. For example, would a patient requiring a 1:1 patient-to-nurse ratio in the morning still need this in the afternoon of the same day? In this perspective, two time windows are particularly of interest: 1) to know how many nurses are needed for the next shift based on the actual shift, which is crucial for nurse rostering, and 2) to predict nurse staffing needed for the same shift the next day, which allows more anticipated planning or modelling of nurse staffing.

Therefore, the aim of this study was to determine the NAS cut-off value which can best distinguish between lower, average and high nurse-level intensity ICU patients as unanimously perceived by care providers. Furthermore, we aimed to evaluate whether these NAS cut-offs allowed to predict care intensity in the next shift, or in the same shift during the next day.

\section{Methods}

\subsection{Study design}

This single-center prospective observational study involved a multicomponent quantitative data collection from six ICUs and three lower acuity units between March 20, 2013, and September 12, 2013. The study was conducted in University Hospitals Leuven, Belgium, a tertiary care center with 1995 beds. The six ICUs have a patient-to-nurse ratio of 2:1. The three lower acuity units have a higher patient-to-nurse ratio, ranging from 3:1 to 5:1.

All patients admitted to these units during the study period were included. All ICU nurses involved in direct patient care, ICU nurse managers and intensive care physicians participated to this study. Physicians or nurse managers who conducted exclusively non-clinical activities during the study time-window did not participate in this study. The aims and design of the study were explained to all participating health care professionals at multiple occasions.

\subsection{Study measures}


To quantify NAS-defined care intensity, the hospital's Patient Data Management System (PDMS) was used. TISS and NAS were introduced upon the implementation of the PDMS in 2006, but their clinical use had not been implemented. For this study, PDMS was reconfigured to provide: (1) items automatically derived by connected devices (e.g. electrocardiogram registrations); (2) therapeutic entries (e.g. arterial line or wound care registration); (3) medical prescriptions performed (e.g. total hydration); (4) monitoring and titration; (5) hygiene procedures; (6) mobilization and positioning of the patient; (7) support and care of relatives/patients; and (8) administrative and management tasks plus medical interventions. The latter 5 items were manually entered at the end of each shift into the PDMS for the purpose of this study. These items generated - on a daily basis and according to published guidelines - one overall day-NAS and one specific shift-NAS per patient (Debergh et al., 2012). Shifts were defined as morning ( $7 \mathrm{am}$ to $14.39 \mathrm{pm}$ ), evening (14.40 pm to $22.19 \mathrm{pm}$ ) and night (22.20pm to 6.59am). The day-NAS was the sum of the maximum scores for each item scored during the specific day.

Patients were categorized into high, average and lower nurse-level intensity as perceived by nurses, nurse managers and physicians, who were all blinded for the actual NAS. During the second half of their shift, nurses completed a care intensity perception survey in which they were asked the following question: "What is your opinion on the workload for nurses caring for this patient?”. Response categories were: (1) High nurse-level intensity, i.e. 'A critically ill patient requiring more attention than a typical ICU patient (requiring a patient-to-nurse ratio of 1:1)'; (2) Average nurse-level intensity, i.e. 'A typical ICU patient (requiring a patient-tonurse ratio of 2:1)'; or (3) Lower nurse-level intensity, i.e. 'A lower intensity patient (requiring a patient-to-nurse ratio of 3:1)'. Nurse managers and physicians were only surveyed once a day during weekdays. Missing data were checked on a daily basis, and non-responders were asked to complete the information as soon as possible, at the latest the next day. For seven out of nine participating units, NAS items were collected for all shifts, in 2 units however, data collection during evening and night shifts was considered unfeasible. The NAS cut-offs were determined based on patient-shifts classified unanimously by nurses, nurse managers and physicians as high, average or lower intensity.

Given their role in providing essential items for the calculation of the NAS (see just above) several nurse characteristics (age, gender, years of experiences, 
employment, and education (advanced bachelor in emergency and intensive care)) were collected. Also, some basic patient characteristics (age, gender, and length of stay) were registered.

\subsection{Statistical analysis}

Data were presented as percentages, means $\pm \mathrm{SD}$ or medians (25th- 75th percentile), and eventually grouped in contingency tables, as appropriate. Data were compared using Student's $t$-test, Chi-square test, and Mann-Whitney U test. Receiver operating characteristic (ROC) analysis is applied to determine the NAS cut-offs that best distinguish between different levels of intensity. The area under a ROC curve (AUC) represents the overall discriminatory ability of the test, where a value of 1.0 denotes perfect ability, and a value of 0.5 denotes no ability. The potential for a given NAS cut-off-based intensity level to predict care intensity during the next shift and during a same shift during the next day, was estimated by logistic regression models. For this purpose, the data set was evenly split into a construction and a "naïve” validation set. In order to avoid over-sampling of longstay patients, no more than 10 consecutive shifts were taken into account in one patient.

Statistical analysis was performed with JMP ${ }^{\circledR}$ SAS software. Copyright $(02016$ SAS Institute Inc. SAS and all other SAS Institute Inc. product or service names are registered trademarks or trademarks of SAS Institute Inc., Cary, NC, USA.

\subsection{Ethical approval}

The study protocol was approved by the Medical Ethics Committee University Hospitals Leuven (ML 8559). Given the observational design of the study, the need for informed consent was waived.

\section{Results}

\subsection{Sample description}

During the study period, a total of 3295 patients were included, of which 1972 admitted to one of six ICUs, and 1323 to one of three lower acuity units. The population was predominantly male (62\%), with a mean age of 59 (SD 22.2) years, and median length of stay of 2.8 days (Q1: 1.1, Q3: 5.9). 
The majority of nurses $(n=412)$ was female $(70.4 \%)$, with a mean age of 41 (SD 11.8) years, and 17.2 (SD 11.9) years of experience. Three out of four nurses (74.0\%) had a specialized degree in intensive and emergence care. There were no relevant differences in demographic characteristics nor level of education of nurses across units.

Nurses provided items allowing the PDMS to calculate 42568 shift-NAS, resulting in 16780 day-NAS. The nurses moreover provided their perception of optimum patient-to-nurse ratio (nurse-level intensity) for 40246 patient-shifts. Twenty-four nurse managers completed 9891 care intensity perception surveys, of which 9751 (95.5\%) could be linked to the NAS-scores for the corresponding shift. Thirtyseven physicians completed 8807 care intensity perception surveys, of which 8713 (98.9\%) could be linked to the corresponding NAS. Indeed, for less than 5\% of surveys no corresponding NAS was available due to missing NAS-items when patients went to the operating theater for a long period of time, were admitted close to shift ends.

\subsection{Nursing activities score}

The average day NAS was 61.5\% (SD 21.1\%). Of the 42568 shift NAS, 15437 were morning shift scores, 13665 evening shift scores, and 13466 night shift scores. Table 1 displays findings for NAS across shifts, and across ICUs and lower acuity care units. Differences across shifts and type of units were observed. To interpret the findings in terms of average workload per nurse, for example, the average day NAS of $65.1 \%$ on ICUs would translate to an average $130.2 \%$ NAS workload per nurse (as default staffing is 2 patients per nurse in these units). As previously mentioned, a NAS workload of $100 \%$ is supposed to consume $100 \%$ of a nurses' time.

Table 1. NAS day, morning, evening and night scores.

\begin{tabular}{lllllll} 
& Overall & & \multicolumn{2}{c}{ Intensive care units } & \multicolumn{2}{c}{ Lower acuity care units } \\
& $\mathbf{n}$ & mean (SD) & N & mean (SD) & n & mean (SD) \\
\hline Day (overall) & 16780 & $61.5 \%(21.1 \%)$ & 12893 & $65.1 \%(19.8 \%)$ & 3887 & $49.6 \%(21.0 \%)$ \\
Morning & 15437 & $55.0 \%(19.6 \%)$ & 11578 & $58.5 \%(18.4 \%)$ & 3859 & $44.4 \%(19.1 \%)$ \\
Evening & 13665 & $55.4 \%(18.0 \%)$ & 12127 & $55.6 \%(17.8 \%)$ & 1538 & $53.1 \%(19.4 \%)$ \\
Night & 13466 & $53.3 \%(16.4 \%)$ & 12001 & $53.6 \%(16.7 \%)$ & 1465 & $51.1 \%(13.2 \%)$
\end{tabular}




\subsection{Differentiation in patient intensity by nurses, nurse managers and physicians}

For 8215 patient-shifts the nurse-level intensity as perceived by both nurses', nurse managers', and physician' was available. Their opinions on patient intensity corresponded in 4693 (57.1\%) cases, 24\% of which were perceived as lower intensity patients, $32 \%$ as average intensity, and $1 \%$ as high intensity

patients. Table 2 illustrates degrees of agreement between healthcare professionals. Nurses and nurse managers identified more patients as high intensity compared to physicians, and agreement among all three groups on high intensity was poor.

Table 2. Degree of agreement in healthcare professionals' perception of care intensity.

\begin{tabular}{|c|c|c|c|}
\hline & $\begin{array}{l}\text { Agreement with } \\
\text { nurses }\end{array}$ & $\begin{array}{l}\text { Agreement with nurse } \\
\text { managers }\end{array}$ & $\begin{array}{l}\text { Agreem } \\
\text { physicic }\end{array}$ \\
\hline \multicolumn{4}{|l|}{ Nurses’ perception } \\
\hline $\begin{array}{l}\text { Lower nurse-level intensity }(n=14 \\
300,36 \%)\end{array}$ & - & $82.3 \%$ & $78.9 \%$ \\
\hline $\begin{array}{l}\text { Average nurse-level intensity } \\
(n=22604,56 \%)\end{array}$ & - & $70.6 \%$ & $74.5 \%$ \\
\hline $\begin{array}{l}\text { High nurse-level intensity } \\
(n=3342,8 \%)\end{array}$ & - & $39.3 \%$ & $17.9 \%$ \\
\hline \multicolumn{4}{|l|}{ Nurse managers' perception } \\
\hline $\begin{array}{l}\text { Lower nurse-level intensity } \\
(n=4179,42 \%)\end{array}$ & $73.0 \%$ & - & $75.2 \%$ \\
\hline $\begin{array}{l}\text { Average nurse-level intensity } \\
(n=4823,49 \%)\end{array}$ & $76.9 \%$ & - & $76.8 \%$ \\
\hline $\begin{array}{l}\text { High nurse-level intensity }(n=889 \text {, } \\
9 \%)\end{array}$ & $40.5 \%$ & - & $18.7 \%$ \\
\hline \multicolumn{4}{|l|}{ Physicians' perception } \\
\hline $\begin{array}{l}\text { Lower nurse-level intensity } \\
(n=3719,42 \%)\end{array}$ & $68.4 \%$ & $73.3 \%$ & - \\
\hline $\begin{array}{l}\text { Average nurse-level intensity } \\
(n=4797,55 \%)\end{array}$ & $73.6 \%$ & $70.1 \%$ & - \\
\hline $\begin{array}{l}\text { High nurse-level intensity }(n=291 \text {, } \\
3 \%)\end{array}$ & $52.2 \%$ & $51.4 \%$ & - \\
\hline
\end{tabular}

The average NAS was 44.2\% (median: 40.7; Q1: 33.9 - Q3: 50.9) for patients perceived as lower intensity, 65.0\% (median: 62.6; Q1: 57.3 - Q3: 72.7) for average intensity and 82.7\% (median: 79.9; Q1: 72.3 - Q3: 90.9) for high intensity patients. Sup. Fig. 1 displays the distribution of the data for lower intensity, average 
intensity and high intensity patients. Mann-Whitney U test showed that differences across groups were all statistically significant $(p<0.0001)$.

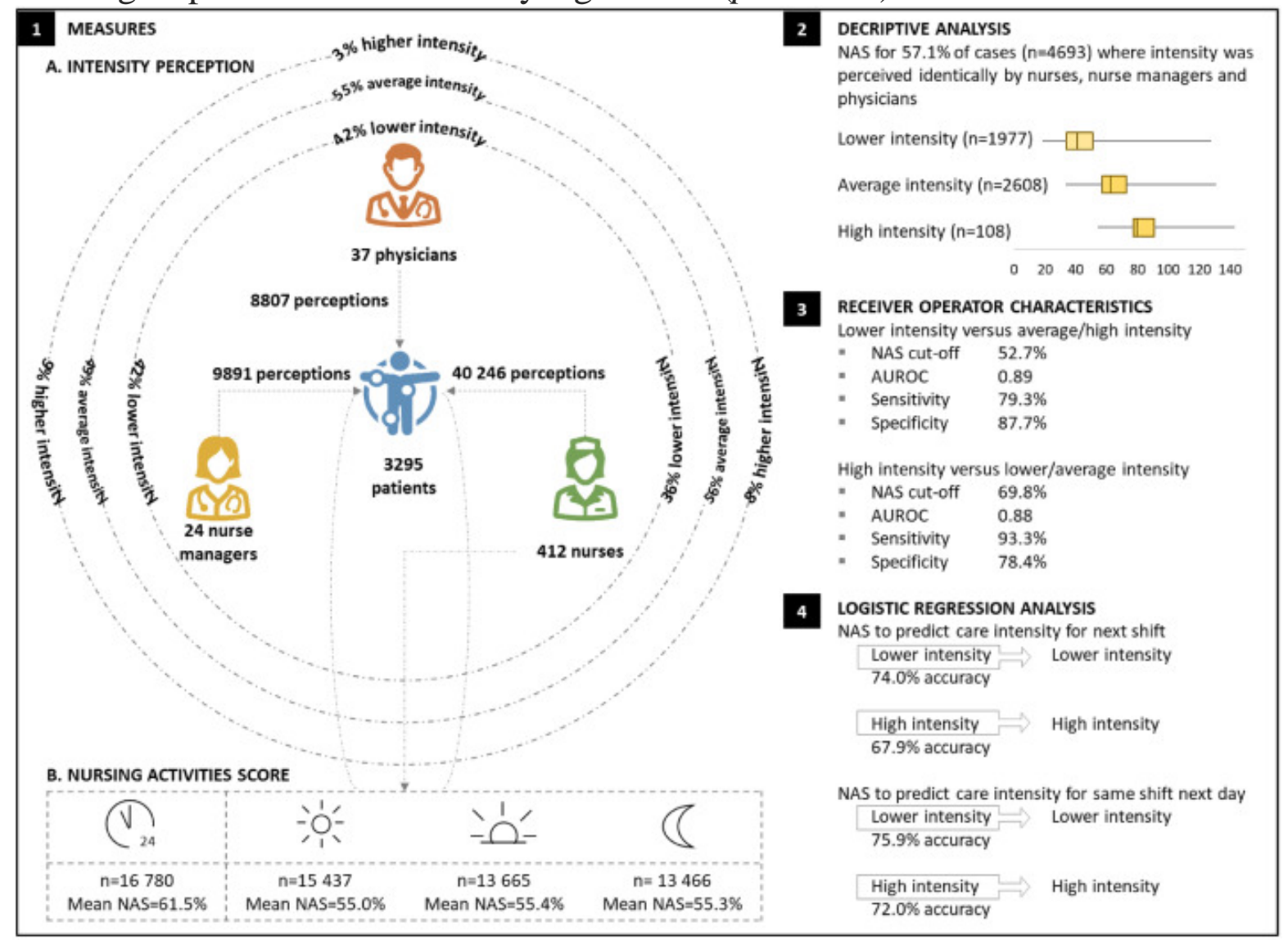

Fig. 1. Summary of data and findings.

\subsection{NAS cut-offs that distinguished between lower intensity patients and other} patients, and between high intensity patients and other patients

ROC analysis for the distinction between lower intensity patients (who can be adequately taken care of in a 3:1 patient to nurse staffing ratio) and other intensity patients (i.e. average and high intensity) provided an AUC of 0.89. The optimal NAS cut-off was smaller than $52.7 \%$, with a sensitivity of $79.3 \%$ and specificity of 87.8\% (Table 3). This cut-off value would classify 39.7\% (14 160 of 35 706) of shift NAS measurements on ICUs as lower intensity, and 68.2\% (4677 of 6862) of shift NAS measurements in lower intensity units.

Table 3. NAS cut-offs to distinguish between lower intensity patients and other patients, and between high intensity patients and other patients.

\begin{tabular}{|lll} 
& $\begin{array}{l}\text { High intensity versus average and low } \\
\text { intensity }\end{array}$ & $\begin{array}{l}\text { Lower intensity versus average and high } \\
\text { intensity }\end{array}$ \\
\hline Area under the curve & 0.88 & 0.89
\end{tabular}




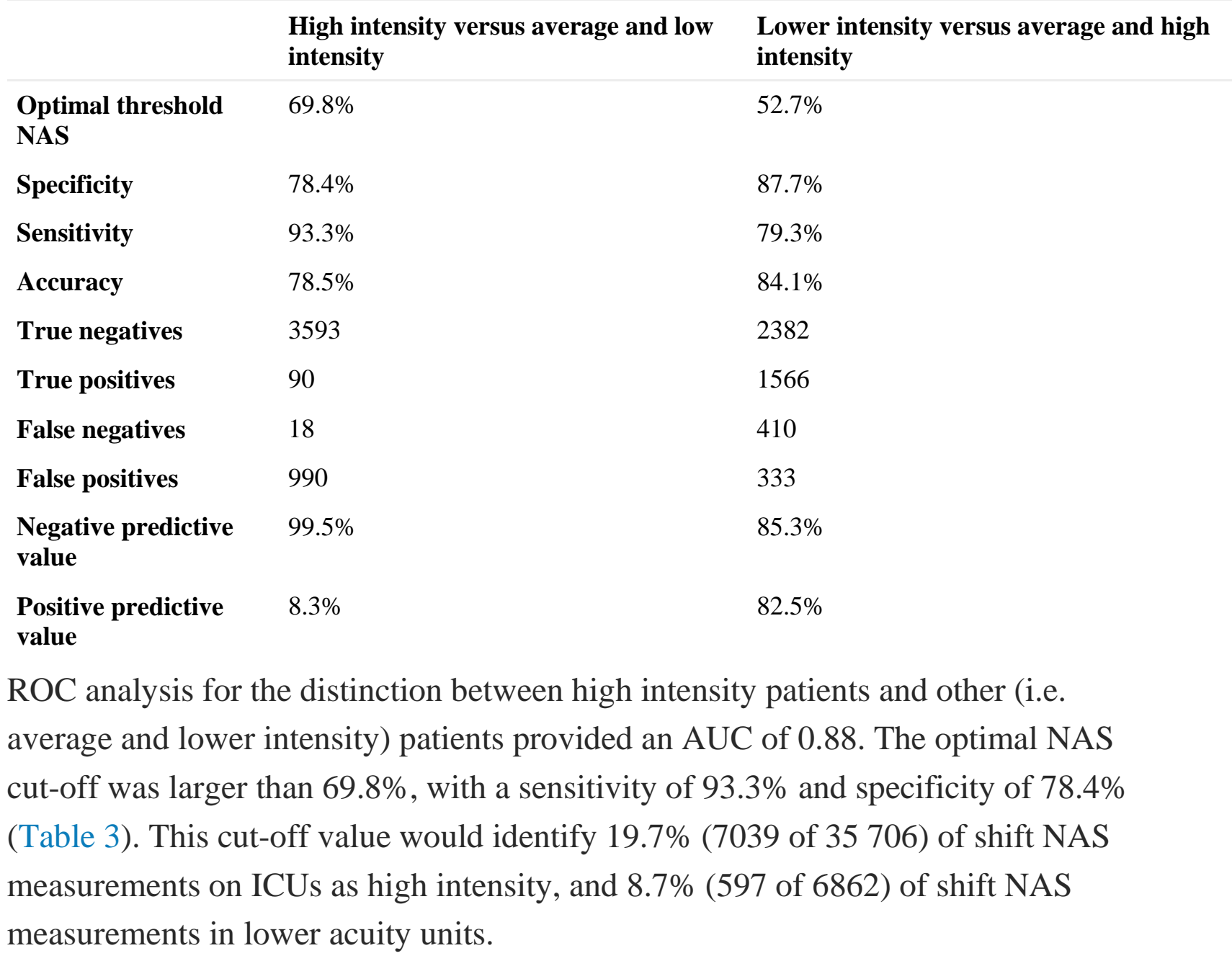

\subsection{NAS cut-offs to predict care intensity in a next shift}

2298 patients, for whom at least one pair of consecutive shift NAS points was available, were randomly split into a development sample (1149 patients and 7657 pairs of current shift and following shift NAS) and validation sample (1149 patients and 7232 pairs). Logistic regression analysis predicting whether the NAS in the consecutive shift would be below the cutoff for lower intensity (52.7\%) showed $74.0 \%$ accuracy (negative predictive value: $77.8 \%$, positive predictive value: 69.8\%) (Table 4). Sup. Fig. 2 panels A and B present the ROC curves for the development and validation sample, respectively.

Table 4. NAS cut-offs to predict care intensity in the next shift and in the same shift the next day. 


\begin{tabular}{lllll} 
& $\begin{array}{l}\text { Below cut-off of } \mathbf{5 3 . 7} \\
\text { for lower intensity }\end{array}$ & $\begin{array}{l}\text { Above cut-off of } \mathbf{6 9 . 8} \\
\text { for high intensity }\end{array}$ & $\begin{array}{l}\text { Below cut-off of } \mathbf{5 3 . 7} \\
\text { for lower intensity }\end{array}$ & $\begin{array}{l}\text { Above cut-off of } 69.8 \\
\text { for high intensity }\end{array}$ \\
\hline $\begin{array}{l}\text { Area under the } \\
\text { curve }\end{array}$ & 0.78 & 0.73 & 0.80 & 0.77 \\
$\begin{array}{l}\text { Number of NAS } \\
\text { pairs }\end{array}$ & 7232 & 7232 & 5035 & 5035 \\
\hline $\begin{array}{l}\text { Specificity } \\
\text { Sensitivity }\end{array}$ & $74.8 \%$ & $67.9 \%$ & $76.5 \%$ & $72.2 \%$ \\
\hline $\begin{array}{l}\text { Accuracy } \\
\text { True negatives }\end{array}$ & $73.0 \%$ & $67.5 \%$ & $75.0 \%$ & $70.8 \%$ \\
True positives & 2341 & $67.9 \%$ & $75.9 \%$ & $72.0 \%$ \\
False negatives & 864 & 4228 & 2236 & 3094 \\
False positives & 1013 & 680 & 1584 & 532 \\
$\begin{array}{l}\text { Negative } \\
\text { predictive value }\end{array}$ & $77.7 \%$ & 327 & 528 & 219 \\
\hline $\begin{array}{l}\text { Positive } \\
\text { predictive value }\end{array}$ & $69.8 \%$ & 1997 & 687 & 1190 \\
\hline
\end{tabular}

Logistic regression analysis predicting whether the NAS in the consecutive shift would be above the cutoff for high intensity (69.8\%) showed 67.9\% accuracy (negative predictive value: 92.8\%, positive predictive value: 25.4\%) (Table 4). Sup. Fig. 2 panels $\mathrm{C}$ and $\mathrm{D}$ present the ROC curves for the development and validation sample, respectively. An average intensity patient was most likely to remain so in the next shift. However, a lower intensity patient had a more than $50 \%$ likelihood to become an average intensity patient in the next shift in case that patient was not discharged. The development and validation samples generated very similar results.

\subsection{NAS cut-offs to predict care intensity on the same shift during the next day}

The random split development sample included 5444 pairs (current shift and same shift the next day) and the validation sample 5035 pairs. Logistic regression analysis predicting whether the NAS in the same shift on the next day would be below the cutoff for lower intensity (52.7\%) showed 75.9\% accuracy (negative predictive value: 80.9\%, positive predictive value: 69.7\%) (Table 4). Sup. Fig. 2 panels $\mathrm{E}$ and $\mathrm{F}$ present the ROC curves for the development and validation sample, respectively. 
Logistic regression analysis predicting whether the NAS in the same shift on the next day would be above the cutoff for high intensity (69.8\%) showed 72.0\% accuracy (negative predictive value: 93.4\%, positive predictive value: $30.9 \%$ ) (Table 4). Sup. Fig. 2 panels $\mathrm{G}$ and $\mathrm{H}$ present the ROC curves for the development and validation sample, respectively.

Fig. 1 summarizes the main data and findings of the study.

\section{Discussion}

Our large PDMS-supported collection of Nursing Activities Score, combined with care providers' perception of nurse-level intensity in different intensive care and lower acuity units in a tertiary hospital, revealed a high nurse workload, exceeding a 100\% NAS particularly for nurses working in 2:1 and 3:1 patient-to-nurse ratio's. Nurses, nurse managers and physicians agreed rather good on the large group of patient-shifts where a 2-1 patient-to-nurse staffing-ratio was deemed adequate. However, unanimity on "high intensity" shifts requiring a patient-to-nurse ratio of 1:1 was rare. Finally, NAS varied considerably between subsequent shifts in a single patient, and also for the same shift in the following days, which may complicate NAS-based planning of nurse allocation.

Daily NAS in our study were high, ranging from on average $49.6 \%$ in lower intensity units to $65.1 \%$ in the ICUs. By consequence, this will result in total NAS for one nurse regularly exceeding $100 \%$ both in 3:1 staffing and 2:1 patient-tonurse staffing. Indeed, given the distribution of the NAS (Fig. 1) it is mathematically impossible to create pairs of average intensity patients or triplets of lower intensity patients not exceeding a NAS of $100 \%$. According to the developers of the NAS, a NAS of $100 \%$ is supposed to consume $100 \%$ of a nurses' shift (Miranda et al., 2003). Given these findings, both the evolution of adverse events and nurse well-being should be closely and continuously monitored. This may be even more the case in the lower intensity units. Excessive workload may be partially prevented by conscientious and dynamic planning of nurse staffing, taking into account summation of NAS per pair or triplet of patients when allocating them to one nurse. This exercise is complicated, however, given the high variability in NAS for the same patient observed on a shift by shift basis.

Agreement between nurses, nurse managers and physicians on which patients require a lower or average staffing ratio was high. However, agreement on high 
intensity patients requiring a 1:1 patient-to-nurse ratio was poor to very poor. Interestingly, nurse managers were more inclined to label patient shifts as high acuity as compared to physicians ( $9 \%$ vs 3\%), yet they still failed to identify those patients who were experienced as high acuity by their bedside colleague nurses. Assuming that none of the clinician groups can be considered the gold standard we built our model based on only those patients where all three disciplines of health care professionals agreed. Therefore, the implementation of NAS cut-offs proposed in the actual paper or of any other tool will ultimately require clinical validation. Such validation goes beyond the caregivers' perception and should consider the impact of a given nurse staffing strategy on clinical outcome for the patients and well-being of the bed-side nurses. It may depend on the broader organization of care and on the case mix within the intensive care unit where such validation would be conducted.

Previous research has shown that the NAS can be used to assess workload per working shift (Debergh et al., 2012). This encouraged us to investigate the ability to take workload into account for manpower planning. Despite the within-patient variability in NAS between shifts, we found relatively good positive and negative predictive values both in models predicting the immediate following shift, as well as the same shift on the next day based on the proposed NAS cut-off for lower acuity patients. This relatively good predictive value, combined with the high rates of agreement (70\% to $80 \%$ overlap) between nurses, nurse managers and physicians in labelling patient-shifts as lower acuity was reassuring. It could allow nurse managers and/or nurse staffing tools to identify a large group of patients (36$42 \%$ of all patients admitted to ICUs and lower acuity units) that could be safely taken care of in a lower staffing ratio in the next shift or day. However, this is not the case for the relatively small proportion of high intensity patients (3-9\% of all patients studied). The agreement between different health care professionals was low, and the models were not performing too well either. This is not surprising in the context of an ICU. The unpredictable clinical evolution in the most severely ill patients is a typical feature of critical illness. Therefore, NAS-based nurse staffing tools should function at least at a (sub) unit level (rather than individual patient level) allowing to safely "absorb” potential mistakes in estimated staffing needs. Likewise, the identification of approximately $30 \%$ of patient shifts in the ICU as "lower intensity", based on our cut-off, was an important and reassuring finding. It 
may constitute some safety buffer for unforeseen high intensity shifts in the near future (Dahl et al., al.).

\subsection{The present study has some limitations}

First, our study was performed in only one Belgium tertiary referral university hospital. Although it validates the concept of a NAS cut-off in predicting nursing workload, these cut-off values themselves cannot be generalized to other ICUs as pathology and therapeutic environment may be very specific and determining for nurse staffing needs. Second, for practical feasibility, only NAS during weekdays were taken into account, as nurse manager and physician scores were not obtained in the weekend and on holidays. This may have influenced our findings, as weekend NAS have been found to be lower (Debergh et al., 2012). Also, for two units, NAS was only measured during the morning shifts. Third, the NAS itself has some limitations. Nursing is not a sum of clearly delineated activities. The simultaneous performance of activities makes it difficult to make a correct assessment of time required to complete these activities (Gonçalves et al., 2007). A face and content re-evaluation was performed in Italy, suggesting that the NAS needs a revision to be in accordance with current practice in ICUs (Palese et al., 2016). Indeed, a number of time-consuming activities are not or insufficiently taken into account in the NAS (e.g. turnover, documentation of care work interruption by telephone calls) (Myny et al., 2012). Also, the NAS questions about care intensity may be prone to subjective interpretation (Vincent and Moreno, 2010). Finally, quality of patient care and the well-being of the health care professionals in an ICU setting is probably determined by more than nurse to patient staffing ratios. For example, the presence of advanced practice nurses has been associated with a reduction in 30-day mortality. The contribution of other health care professionals, not in the least intensive care physicians, is also well documented. For example, a large UK retrospective cohort study found that hospital mortality is lowest at a patient-to-intensive care physician ratio of 7.5 (Gershengorn et al., 2017). Likewise, Neuraz et al. found that an insufficient patient-to-physician ratio above an observed maximum threshold of 14 was associated with an increased risk of mortality (Neuraz et al., 2015). The College of Intensive Care Medicine of Australia recommends an intensive care physician-to-patient ratio of 1 per 8-15 patients (College of Intensive Care Medicine of Australia and New Zealand 2011). Also allied health professionals contribute to safe patient care in the ICU (Knight, 
2016). A large percentage of nurses and physicians perceiving care as inappropriate, identified good teamwork, mandatory family meetings, and formal communication training as potential solutions (Piers et al., 2014; Anstey et al., 2015; Piers et al., 2011).

\section{Conclusions}

This large observational prospective study on workload, as perceived by healthcare professionals and NAS at a patient-shift level, revealed that NAS cut-offs could contribute considerably in predicting patient-to-nurse staffing ratios in the next shift or day. However, quality of care and the safety of patients and nurses is determined by far more than nurse staffing and workload alone. Severity of illness can change very quickly, and identification or prediction of high nurse-level intensity patient-shifts appears most complicated. Therefore, nurse managers or nurse staffing tools should integrate additional parameters and incorporate buffers in order to safely absorb unavoidable mistakes.

\section{Funding}

No external funding

\section{CRediT authorship contribution statement}

Karen Decock: Conceptualization, Methodology, Investigation, Data curation, Formal analysis, Writing - original draft. Michael P. Casaer: Conceptualization, Methodology, Investigation, Writing - original draft, Project administration, Supervision. Fabian Guïza: Methodology, Formal analysis, Data curation, Visualization, Writing - original draft. Pieter Wouters: Software, Data curation, Investigation, Writing - review \& editing. Mieke Florquin: Investigation, Writing - review \& editing. Alexander Wilmer: Investigation, Writing - review \& editing. Stefan Janssens: Investigation, Writing - review \& editing. Sandra Verelst: Investigation, Writing - review \& editing. Greet Van den Berghe: Conceptualization, Investigation, Writing - original draft, Project administration, Supervision. Luk Bruyneel: Methodology, Visualization, Writing original draft, Supervision.

\section{Declaration of Competing Interest}




\section{References}

\section{Rothberg et al., 2005}

M.B. Rothberg, I. Abraham, P.K. Lindenauer, D.N Rose

Improving nurse-to-patient staffing ratios as a cost-effective safety intervention Med. Care, 43 (2005), pp. 785-791

[cited 2014 Dec 9]

\section{Needleman et al., 2006}

J. Needleman, P.I. Buerhaus, M. Stewart, K. Zelevinsky, S Mattke

Nurse staffing in hospitals: is there a business case for quality?

Health Aff. (Millwood), 25 (2006), pp. 204-211

[cited 2014 Apr 7]

\section{Aiken et al., 2014}

L.H. Aiken, D.M. Sloane, L. Bruyneel, K. Van den Heede, P. Griffiths, R. Busse, et al.

Nurse staffing and education and hospital mortality in nine European countries: a retrospective observational study

Lancet, 383 (2014), pp. 1824-1830

[cited 2014 Jul 9]

Kane et al., 2007

R.L. Kane, T.A. Shamliyan, C. Mueller, S. Duval, T.J Wilt

The association of registered nurse staffing levels and patient outcomes: systematic review and meta-analysis

Med Care, 45 (2007), pp. 1195-1204

[cited 2014 Apr 10]

Aragon Penoyer, 2010

D. Aragon Penoyer

Nurse staffing and patient outcomes in critical care: a concise review Crit. Care Med., 38 (2010), pp. 1521-1528

[cited 2017 Mar 1]

\section{Numata et al., 2006}

Y. Numata, M. Schulzer, R. van der Wal, J. Globerman, P. Semeniuk, E. Balka, et al.

Nurse staffing levels and hospital mortality in critical care settings: literature review and meta-analysis

J. Adv. Nurs., 55 (2006), pp. 435-448

[cited 2017 Mar 1] 
E. West, N. Mays, A.M. Rafferty, K. Rowan, C Sanderson

Nursing resources and patient outcomes in intensive care: a systematic review of the literature

Int. J. Nurs. Stud., 46 (2009), pp. 993-1011

[cited 2017 Mar 1]

McGahan et al., 2012

M. McGahan, G. Kucharski, F. Coyer

Winner ACCCN Best Nursing Review Paper 2011 sponsored by Elsevier. Nurse staffing levels and the incidence of mortality and morbidity in the adult intensive care unit: a literature review

Aust. Crit. Care, 25 (2012), pp. 64-77

[cited 2017 Oct 4]

West et al., 2014

E. West, D.N. Barron, D. Harrison, A.M. Rafferty, K. Rowan, C Sanderson

Nurse staffing, medical staffing and mortality in Intensive Care: an observational study

Int J. Nurs. Stud., 51 (2014), pp. 781-794

Neuraz et al., 2015

A. Neuraz, C. Guérin, C. Payet, S. Polazzi, F. Aubrun, F. Dailler, et al.

Patient mortality is associated with staff resources and workload in the ICU: a multicenter observational study

Crit. Care Med., 43 (2015), pp. 1587-1594

[cited 2017 Oct 11]

\section{Coffman et al., 2002}

J.M. Coffman, J.A. Seago, J Spetz

Minimum nurse-to-patient ratios in acute care hospitals in California Health Aff. (Millwood), 21 (2002), pp. 53-64

[cited 2014 Dec 9]

\section{American Nurses Association 2015}

American Nurses Association. State legislative agenda on nurse staffing. 2015.

Available from:http://www.nursingworld.org/MainMenuCategories/Policy-

Advocacy/State/Legislative-Agenda-Reports/State-StaffingPlansRatios

College of Intensive Care Medicine of Australia and New Zealand 2011

College of Intensive Care Medicine of Australia and New Zealand. Minimum

Standards for Intensive Care Units Policy. 2011.

\section{Federal Public Service Health Food Chain Safety and Environment 1998}

Federal Public Service Health Food Chain Safety and Environment. Royal Decree of

27 April 1998 regarding the requirements for intensive care units. 1998. 
P. Kiekkas, G.C. Sakellaropoulos, H. Brokalaki, E. Manolis, A. Samios, C. Skartsani, et al.

Association between nursing workload and mortality of intensive care unit patients

J. Nurs. Scholarsh., 40 (2008), pp. 385-390

[cited 2017 Feb 28]

Cullen et al., 2020

D.J. Cullen, J.M. Civetta, B.A. Briggs, L.C Ferrara

Therapeutic intervention scoring system: a method for quantitative comparison of patient care

Crit. Care Med., 2 (2020), pp. 57-60

[cited 2017 Feb 28]

Keene and Cullen, 1983

Keene A.R., Cullen D.J.

Therapeutic Intervention Scoring System: update 1983. Crit. Care Med.. 1983[cited 2017 Oct 11];11:1-3.

Miranda et al., 1996

D.R. Miranda, A. de Rijk, W Schaufeli

Simplified Therapeutic Intervention Scoring System: the TISS-28 items-results from a multicenter study

Crit. Care Med., 24 (1996), pp. 64-73

[cited 2017 Feb 28]

Lee et al., 2017

A. Lee, Y.S.L. Cheung, G.M. Joynt, C.C.H. Leung, W.-.T. Wong, C.D Gomersall

Are high nurse workload/staffing ratios associated with decreased survival in critically ill patients? A cohort study

Ann. Intensive Care. Springer Paris, 7 (2017), p. 46

[cited 2017 Oct 11]

Miranda et al., 2003

D.R. Miranda, R. Nap, A. de Rijk, W. Schaufeli, G. Iapichino, TISS Working Group

Therapeutic Intervention Scoring System. Nursing activities score

Crit. Care Med., 31 (2003), pp. 374-382

[cited 2017 Mar 1]

Padilha et al., 2010

K.G. Padilha, R.M.C. de

Sousa, P.C. Garcia, S.T. Bento, E.M. Finardi, R.H.K Hatarashi 
Nursing workload and staff allocation in an intensive care unit: a pilot study according to Nursing Activities Score (NAS)

Intensive Crit. Care Nurs., 26 (2010), pp. 108-113

[cited 2017 Mar 1]

\section{Padilha et al., 2015}

Padilha K.G., Stafseth S., Solms D., Hoogendoom M., Monge F.J.C., Gomaa O.H., et al.

Nursing Activities Score: an updated guideline for its application in the Intensive Care Unit. Rev da Esc Enferm da USP. 2015[cited 2017 Mar 1];49:131-7.

Gonçalves et al., 2007

L.A. Gonçalves, K.G. Padilha, R.M Cardoso Sousa

Nursing activities score (NAS): a proposal for practical application in intensive care units

Intensive Crit. Care Nurs., 23 (2007), pp. 355-361

[cited 2017 Mar 1]

Ferreira et al., 2017

P.C. Ferreira, R.C. Machado, Q.C.S. Martins, S.F Sampaio

Classificação de pacientes e carga de trabalho de enfermagem em terapia intensiva: comparação entre instrumentos

Rev. Gaúcha Enferm., 38 (2017), p. e62782

[cited 2017 Oct 4]

\section{Macedo et al., 2016}

A.P.M. Macedo, C. de, C.M.F.S. Mendes, Candeias, A.L.S. Sousa, M.P.R. Hoffmeiste r, L.V. Lage MIGS

Validação do Nursing Activities Score em unidades de cuidados intensivos portuguesas

Rev. Bras. Enferm., 69 (2016), pp. 881-887

[cited 2017 Oct 4]

Padilha et al., 2008

K.G. Padilha, R.M.C. de Sousa, A.F. Queijo, A.M. Mendes, D.R Miranda

Nursing Activities Score in the intensive care unit: analysis of the related factors Intensive Crit. Care Nurs., 24 (2008), pp. 197-204

[cited 2017 Mar 1]

\section{Debergh et al., 2012}

D.P. Debergh, D. Myny, I. Van Herzeele, G. Van Maele, D.R. Miranda, F Colardyn

Measuring the nursing workload per shift in the ICU

Intensive Care Med., 38 (2012), pp. 1438-1444

[cited 2017 Mar 1] 


\section{Dahl et al}

Dahl D., Wojtal G.G., Breslow M.J., Holl R., Huguez D., Stone D., et al. The high cost of low-intensity ICU outliers. J. Healthc. Manag.. [cited 2017 Oct 12];57:421-33; discussion 434.

Palese et al., 2016

A. Palese, I. Comisso, M. Burra, P.P. DiTaranto, L. Peressoni, E. Mattiussi, et al.

Nursing Activity Score for estimating nursing care need in intensive care units: findings from a face and content validity study

J. Nurs. Manag., 24 (2016), pp. 549-559

[cited 2017 Mar 2]

Myny et al., 2012

D. Myny, A. Van Hecke, D. De Bacquer, S. Verhaeghe, M. Gobert, T. Defloor, et al.

Determining a set of measurable and relevant factors affecting nursing workload in the acute care hospital setting: a cross-sectional study

Int. J. Nurs. Stud., 49 (2012), pp. 427-436

[cited 2017 Oct 13]

Vincent and Moreno, 2010

J.-.L. Vincent, R Moreno

Clinical review: scoring systems in the critically ill

Crit Care. BioMed. Central, 14 (2010), p. 207

[cited 2017 Oct 13]

\section{Gershengorn et al., 2017}

H.B. Gershengorn, D.A. Harrison, A. Garland, M.E. Wilcox, K.M. Rowan, H Wunsch

Association of Intensive Care Unit Patient-to-Intensivist Ratios with Hospital

Mortality, 177, JAMA Intern Med. American Medical Association (2017), p. 388

[cited 2017 Oct 11]

Knight, 2016

L.D Knight

Staffing ratios and patient outcomes: looking beyond nurses, doctors and survival at ICU discharge

Crit. Care Med., 44 (2016), pp. e107-e108

[cited 2017 Oct 11]

Piers et al., 2014

R.D. Piers, E. Azoulay, B. Ricou, F. DeKeyser Ganz, A. Max, A. Michalsen, et al.

Inappropriate care in European ICUs

Chest, 146 (2014), pp. 267-275

[cited 2017 Oct 12] 
Anstey et al., 2015

M.H. Anstey, J.L. Adams, E.A McGlynn

Perceptions of the appropriateness of care in California adult intensive care units Crit. Care. BioMed. Central, 19 (2015), p. 51

[cited 2017 Oct 12]

Piers et al., 2011

R.D. Piers, E. Azoulay, B. Ricou, F. Dekeyser Ganz, J. Decruyenaere, A. Max, et al.

Perceptions of Appropriateness of Care Among European and Israeli Intensive Care Unit Nurses and Physicians

JAMA, 306 (2011), p. 2694

[cited 2017 Oct 12] 